\title{
BMJ Open Post-traumatic stress reactions and doctor-certified sick leave after a workplace terrorist attack: Norwegian cohort study
}

\author{
Maria Teresa Grønning Dale (D) , ${ }^{1,2}$ Alexander Nissen (D , ${ }^{1}$ Mona Berthelsen, ${ }^{1}$ \\ Trond Heir ${ }^{1,3}$
}

To cite: Dale MTG, Nissen A, Berthelsen M, et al. Posttraumatic stress reactions and doctor-certified sick leave after a workplace terrorist attack: Norwegian cohort study. BMJ Open 2020;10:e032693. doi:10.1136/ bmjopen-2019-032693

- Prepublication history for this paper is available online. To view these files, please visit the journal online ().

Received 01 July 2019

Revised 24 September 2019

Accepted 28 January 2020

Check for updates

(C) Author(s) (or their employer(s)) 2020. Re-use permitted under CC BY-NC. No commercial re-use. See rights and permissions. Published by BMJ.

${ }^{1}$ Section for Trauma, catastrophes and forced migration, Norwegian Center for Violence and Traumatic Stress Studies, Oslo, Nydalen, Norway ${ }^{2}$ Department of Psychology, University of Oslo, Oslo, Norway ${ }^{3}$ Institute of Clinical Medicine, University of Oslo, Faculty of Medicine, Oslo, Norway

Correspondence to

Dr Maria Teresa Grønning Dale; m.t.g.dale@nkvts.no

\section{ABSTRACT}

Objective To explore the association between the psychosocial work environment and the risk of sick leave among governmental employees with symptom-defined post-traumatic stress disorder (PTSD) after a workplace bomb attack.

Design A prospective study on employees who met the symptom criteria for PTSD. Questionnaire data on the psychosocial work environment 10 months after the terrorist attack was linked to registry data on doctorcertified sick leave in the period 12-22 months after the attack.

Setting The bombing of the government ministries in Oslo, Norway, 22 July 2011.

Participants The study sample consists of 94 Norwegian governmental employees, all with symptom-defined PTSD from the Norwegian version of the PTSD checklist (Posttraumatic Stress Disorder Checklist-Specific) measured 10 months after the attack.

Results After adjustment for sex and severity of PTSD symptoms, predictability at work reduced the odds of sick leave (adjusted OR=0.62, $95 \% \mathrm{Cl} 0.40$ to 0.98 ). Sense of control over decisions at work was associated with fewer absence days for employees with sick leave (adjusted rate ratio $=0.61,95 \% \mathrm{Cl} 0.38$ to 0.98 ).

Conclusions Employees with PTSD after workplace terrorism would benefit from control over their workplace conditions and increased predictability to reduce the risk of sick leave. The findings suggest that the work environment can facilitate employees' work ability after stressful events, independent of severity of PTSD symptoms.

\section{INTRODUCTION}

Post-traumatic stress disorder (PTSD) influences a person's ability to function well at work and in life in general. ${ }^{1-3}$ When PTSD is caused by a workplace-targeted terrorist attack, a common response strategy is avoidance of the workplace. ${ }^{4}$ Consequently, such an attack might contribute to increased risk of sick leave. Still, research on sick leave in relation to PTSD caused by terror is scarce. ${ }^{5}$ There are two relevant studies on workplace terror and sick leave risk. Osinubi et al

\section{Strengths and limitations of this study}

- The psychosocial work environment was assessed before sick leave in the aftermath of a traumatic event.

- For the first time, registry data on doctor-certified sick leave were used in a sampled population with symptom-defined post-traumatic stress disorder (PTSD).

- In terms of generalisability, the study sample consisted of a majority of highly educated government officials and bureaucrats.

- Symptom-defined PTSD and psychosocial work environment relies on self-reported data.

- A small study sample may reduce the statistical power.

found no significant impact on self-reported sick leave and job performance 2 years after the World Trade Center (WTC) terrorist attacks, though $9 \%$ of the workers reported onset of a psychiatric diagnosis in the same period. ${ }^{6}$ In contrast, a study from the Oslo terror bombing based on registry data on sick leave found more than $30 \%$ higher sick leave during the 2 years following the attack compared with the year before the attack. ${ }^{7}$ Another important finding was that sick leave in directly exposed employees returned to pre-attack levels by year three, although the prevalence of PTSD in this group was 17 percent at the same time. This suggests that many employees with PTSD managed to work despite a high symptom load. Further research is needed to explore potential protective and risk factors that could explain why certain employees with likely PTSD manage to work, while others do not.

Psychosocial working conditions are significant for employees' job satisfaction, mental health and sick leave risk, ${ }^{8-10}$ and may therefore moderate the effects of a trauma. 
Prior studies, including a systematic review and a metaanalysis, emphasise the importance of control at work, such as high degree of role clarity, control over work pace and decision-making, and clear and liable management. ${ }^{9-12}$ Both external and internal loci of control can be important, as there seems to be an association between people's sense of control over their own work and improved job satisfaction, performance and work attendance. ${ }^{11}$ The aforementioned studies give us reason to expect that work environments with a high degree of control and predictability are advantageous after experiencing totally unpredictable and threatening workplace violence. However, the role of the work environment in enabling affected workers to return to work and avoid sick leave has rarely been examined empirically. The aim of the present study was therefore to examine whether psychosocial work conditions influence the risk and length of sick leave among employees with symptomdefined PTSD after a terror attack.

\section{METHODS}

\section{Study population and data sources}

This prospective cohort study includes web-based survey data from ministerial employees in 14 of 17 ministries, after a car bomb attack at the Norwegian government offices in Oslo on 22 July 2011. The terror bombing caused substantial damage on buildings and infrastructure, killing eight and injuring 209 people. Previous studies of governmental employees report elevated risk of psychological and somatic health adversities. ${ }^{1314}$ These negative health reactions were found among people who were directly and indirectly exposed..$^{13} 15$ The estimated overall prevalence of post-traumatic stress disorder (PTSD) was 24\% among directly exposed governmental employees and $4 \%$ among those indirectly exposed, signifying that being present at work during the bomb attack was not the only precondition for reporting PTSD symptoms. Common for the employees developing PTSD symptoms are that they all were the explicit target of the terror attack. ${ }^{16}$ It is reasonable to expect that even those who were indirectly exposed and evaluated their life as being in serious danger may develop PTSD. ${ }^{4} 1718$ The present study therefore includes all employees with symptom-defined PTSD, whether indirectly or directly exposed.

The Norwegian Centre for Violence and Traumatic Stress Studies conducted the survey in collaboration with the National Institute of Occupational Health in Norway 10 months after the terrorist attack (T1). Eligible participants were informed about the study through their ministries and received an invitation letter containing a unique $\log$-in code to access the web-based survey, including information on withdrawal procedures. All employees provided written consent, and strict procedures were followed to ensure confidentiality. Fifty-six per cent of the 3520 invited employees consented to participate, and 34 were excluded from the data file due to missing data, leaving us with 1974 employees. In addition, $84 \%$ consented to data linkage on sick leave, and our study population totaled 1649 participants. Data on doctor-certified sick leave was obtained from Statistics Norway and the Norwegian Labour and Welfare Administration.

For the purpose of this study, we used survey data on employees' health and PTSD symptoms, background variables and information on psychosocial work environment from 10 months after the attack, linked with registry data on doctor-certified sick leave for the period of 12-24 months after the attack. Since the first years after the attack were most prominent for sick leave risk and PTSD, we focused on this especially sensitive period. ${ }^{7}$ In this study population $(\mathrm{n}=1649)$, we identified 94 participants eligible for this study, all fulfilling the PTSD symptom criteria and with relevant data on doctorcertified sick leave. Based on the personal identification number from the Norwegian Population register, Statistics Norway performed data linkage and deidentification. The research team did not have access to the key to match data nor the identity behind survey responses. Further details on design and participants have previously been described in a recent article from our research group. ${ }^{7}$ See figure 1 for more information.

\section{Patient and public involvement}

There is no direct patient involvement in this study. Neither patients nor public were directly involved in the development, design or recruitment of the study.

\section{Classification of PTSD}

The Norwegian version of the Post-traumatic Stress Disorder Checklist-Specific (PCL-S) was used to assess symptoms of PTSD. ${ }^{19}$ In this version, the questions were specifically linked to the bomb attack in Oslo on 22 July 2011. All participants were asked to rate the extent to which they had experienced PTSD symptoms during the last 4 weeks and were instructed to answer with reference to the terrorist attack. The PCL-S consists of 17 -items measuring the full domain of PTSD symptom severity from the Diagnostic and Statistical Manual of Mental Disorders, Fourth Edition (DSM-IV). The validated Norwegian version has demonstrated good psychometric and diagnostic properties. ${ }^{20}$ The PTSD diagnosis criteria followed the procedure of positive items required in the three clusters in the DSM-IV system: one positive score in symptom cluster B (re-experiencing), three positive scores in cluster $\mathrm{C}$ (avoidance) and two positive scores in cluster D (hyperarousal). ${ }^{21}$ The A criterion (stressor exposure) was not included in the assessment. All items were scored on an ordinal 5 -point scale from ' $1=$ not at all' to ' $5=$ extremely'. As recommended by the National Center for Post-Traumatic Stress Disorder, an item score of 3 or above was defined as positive for PTSD.$^{19}$ Chronbach's alpha for PCL-S was 0.94 . 


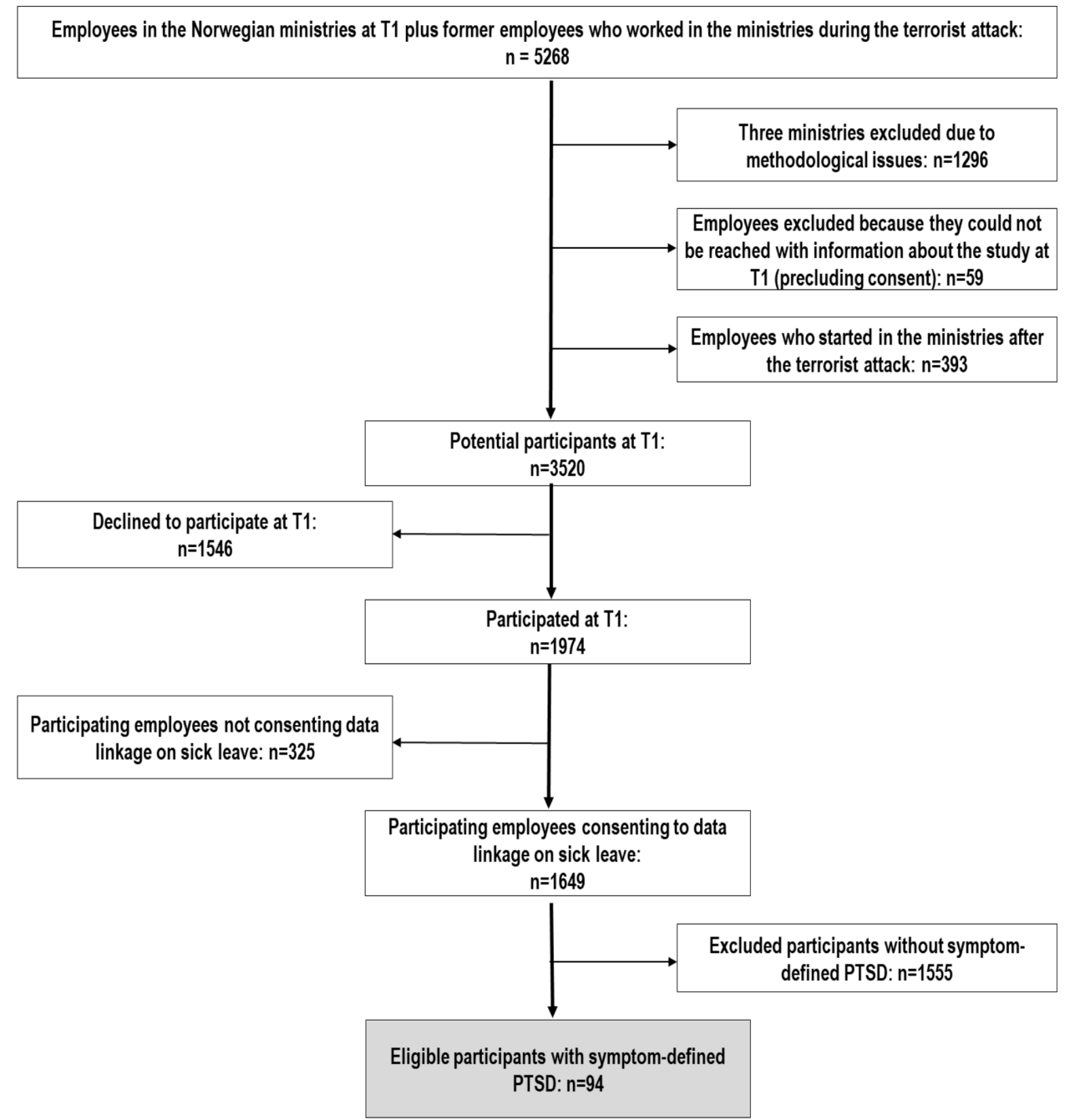

Figure 1 Flowchart displaying participant disposition 10 months after the attack (T1). PTSD, post-traumatic stress disorder.

\section{Sick leave}

Sick leave was measured in the 1-year period after the participants completed the survey 10 months after the attack. This 1-year period consisted of the third and fourth quarter of 2012, and the first and second quarter of 2013. The total number of sick leave days for each participant was weighted by the percentage of full-time employment and the percentage of full-time sick leave. The number of workdays was weighted by percentage of full-time employment, weekends, public holidays and vacations.

\section{Psychosocial work exposures}

Job demands, control at work, predictability, social interactions and organisational culture were measured by the General Nordic Questionnaire for Psychological and Social Factors at Work. ${ }^{22}$ Reliability tests of these scales have demonstrated acceptable internal consistency, with Chronbach's alpha ranging from 0.68 to 0.87 and testretest reliability from 0.66 to 0.83 with more than 5 weeks' interval. ${ }^{23} \mathrm{Job}$ demands were measured by three scales: quantitative demands comprising four items, decision demands with three items and learning demands with three items. A typical item for job demand was 'Do you have too much to do?' Three scales measured control at work: positive challenge comprising three items, control of decisions with five items and control of work pacing with four items. A typical item for control at work was 'Can you set your own work pace?' Predictability at work was measured by the scale long-term predictability (2years) comprising three items. A typical item was 'Do you know what is required in order for you to get a job that you consider attractive in 2 years?' Social interactions were measured by two scales: support from superior comprising three items and support from coworkers with two items. A typical item for support was 'If needed, can you get support and help with your work from your immediate superior?' Finally, organisational culture was measured by three scales: social climate, innovative climate and human resource primacy, all comprising three items. A typical item was 'Are workers well taken care of in your organisation?' All responses were scored on an ordinal 5-point scale ranging from ' $1=$ very seldom or never' to ' $5=$ very often or always', and missing response to one of the items comprising each scale was allowed. 
Table 1 Characteristics of ministerial employees with symptom-defined PTSD and sick leave compared with employees with symptom-defined PTSD without sick leave, 10 months after a workplace terrorist attack on 22 July in Oslo, 2011

\begin{tabular}{|c|c|c|c|c|}
\hline Characteristics & $\begin{array}{l}\text { PTSD without sick } \\
\text { leave }(n=39)\end{array}$ & $\begin{array}{l}\text { PTSD and sick } \\
\text { leave }(n=55)\end{array}$ & $\chi^{2} / \mathbf{F}$ & P value* \\
\hline Females, n (\%) & $23(59.0)$ & $47(85.5) \dagger$ & 8.4 & $<0.01$ \\
\hline$<13$ & 4 (10.3) & $8(14.6)$ & & \\
\hline $13-16$ & $10(25.6)$ & $24(43.6)$ & & \\
\hline Married/cohabiting, n (\%) & $25(64.1)$ & $34(63.0)$ & 0.0 & 0.91 \\
\hline Directly exposed, n (\%) & $15(38.5)$ & $26(47.3)$ & 0.7 & 0.40 \\
\hline Psychological distress, score 1-4 (M \pm SD) & $1.9 \pm 0.8$ & $2.1 \pm 0.9$ & 1.9 & 0.17 \\
\hline Event centrality, score 1-5 (M \pm SD) & $3.2 \pm 1.0$ & $3.4 \pm 0.8$ & 1.4 & 0.24 \\
\hline
\end{tabular}

Case numbers and within-group percentages are shown.

${ }^{*} \mathrm{P}$ values were calculated using analysis of variance for continuous variables and $\chi^{2}$ test for categorical variables. †Differs significantly from those without sick leave.

M, mean; PCL, Post-traumatic Stress Disorder Checklist; PTSD, post-traumatic stress disorder.

\section{Background variables}

The background variables (table 1), including sex, age, educational level, relationship status, directly and indirectly exposed, psychological distress, event centrality, life satisfaction and social support, were retrieved from the questionnaire survey completed 10 months after the terror attack. Education was measured by three levels of education: 'less than 13 years', '13-16 years' and 'more than 16 years'. Relationship status was dichotomised as 'married or cohabiting' and 'single or widow'. Proximity to the explosion was measured by asking participants about their whereabouts at the time of the bomb attack, and responses were dichotomised as 'being present in the government district' and 'not in the government district'.

Psychological distress was measured by the Hopkins Symptom Checklist-10 (SCL-10), with four response categories from ' $1=$ not bothered' to ' $4=$ very bothered'. ${ }^{24} \mathrm{~A}$ typical item was 'worrying too much about things'. The index was scored as the mean of the item score, where an average score above 1.85 is regarded as a valid predictor of depression. Chronbach's alpha for SCL-10 was 0.92. The Centrality of Event Scale (CES) measures to what degree an event was integrated into an individual's life story and identity. ${ }^{25}$ Responses were categorised from ' $1=$ strongly disagree' to ' $5=$ strongly agree' and were calculated as the mean of all items. A typical item was 'I feel that the event has become part of my identity'. Chronbach's alpha for CES was 0.92. Life satisfaction was measured with the oneitem Cantril's Ladder of Life Scale, where overall life satisfaction was rated on a 10-point scale representing steps in the ladder. ${ }^{26} \mathrm{~A}$ score of 10 represents the best possible life you can imagine, and a score of 0 is the bottom step, representing the worst possible life. Finally, social support was measured with the four item Crisis Support Scale (CSS), with response categories ranging from ' $1=$ never' to '7=always'. A typical item was 'How often is someone willing to listen? Chronbach's alpha for CSS was $0.89{ }^{27}$

\section{Covariates}

Covariates that could potentially influence the association between the psychosocial work environment and sick leave risk were considered as potential confounders. The following covariates were included in the main analysis: sex and symptoms of PTSD. Due to a small sample size, we chose a parsimonious structure, adjusting for the most important covariates in the analysis to retain an acceptable number of cases and to be able to detect significant differences between our two groups. We did a preliminary analysis adjusting for education, but that did not change our risk estimates. Therefore, the two most significant variables shown to differ between the groups were entered as covariates in the analysis. See table 1 for information on background variables.

\section{Statistical analysis}

The outcome variable in the present study, sick leave days, is a count variable with overdispersion and an excess of zeros. We therefore used two-part hurdle models to explore sick leave according to various psychosocial work environment factors. ${ }^{28}$ In the first part, we collapsed the outcome variable into two categories: no sick leave (zero count) and sick leave (above zero count). We then estimated the odds of sick leave versus no sick leave for various predictor values and summarised 
this as ORs (95\% CIs) per one unit increase in a given predictor using the lowest value as the reference. In the second part, we used zero-truncated negative binominal regression for the positive counts (ie, those with sick leave) to estimate the length of sick leave for various predictor values. ${ }^{29}$ We summarised this as a count or rate ratio (RR, 95\% CIs) using the lowest predictor value as the reference. To account for varying workdays, as not all employees worked full time during the observation period, the hurdle models were offset by registered workdays in the relevant period.

The binomial regression analyses and the zerotruncated negative binominal analysis were computed in R (The R Foundation for Statistical Computing, Vienna Austria, 2018), with the R packages pscl for hurdle regression (R Development Core Team, 2017) and in STATA V.15.

\section{Sensitivity analysis}

In this study, sick leave prior to the attack is a covariate that can act as a confounder. If sick leave before the attack increases the risk of sick leave after the attack, then our estimated relative risks measuring the association between work factors and sick leave would most likely be attenuated. To capture uncontrolled confounding or systematic change of behaviour due to previous sick leave, we conducted sensitivity analysis by adjusting for previous sick leave prior to the attack, defined as sickness absence days from the first quarter of 2008 up until and including the second quarter of 2011 divided by the number of expected workdays for this period.

\section{RESULTS}

\section{Characteristics of the study population}

In this dataset, the prevalence of PTSD among participants was $57 / 1000(94 / 1649)$ after exclusion of employees not consenting to data linkage on sick leave data $(n=325)$. See figure 1 for more information on participants eligible for this study. In our sample of 55 participants with symptomdefined PTSD and sick leave, the overall mean number of sick leave days was 42 (95\% CI 29.23 to 54.41), while the mean number of registered workdays was 200 in our sample.

Table 1 compares participants with and without sick leave. Both groups have symptom-defined PTSD, and the group with sick leave have higher PTSD scores than the group without sick leave $(p=0.006)$. Women were overrepresented among participants with sick leave; $86 \%$ of the participants with sick leave were female, while $59 \%$ of participants without sick leave were female $(\mathrm{p}=0.004)$. Participants with sick leave did not differ significantly from participants without sick leave with regard to age, marital status, direct versus indirect exposure, psychological distress, event centrality, life satisfaction and social support.

\section{Psychosocial working conditions and sick leave risk}

We observed that predictability at work was associated with reduced odds of sick leave (adjusted OR $(\mathrm{aOR})=0.62$, $95 \%$ CI 0.40 to 0.98 ) independent of symptom-defined PTSD and sex. Other psychosocial working conditions such as job demands, social interactions and organisational culture were not associated with lower odds of sick leave risk when having PTSD. Moreover, the hurdle model estimated the frequency and duration of sick leave (RR). Increased levels of control over decisions at work were associated with fewer sick leave days (adjusted rate ratio $(\mathrm{aRR})=0.61,95 \%$ CI 0.38 to 0.98 ). Other psychosocial working conditions such as job demands, predictability and social interactions were not associated with lower duration of sick leave among employees with PTSD. Organisational cultures such as human resource primacy climate $(\mathrm{aRR}=0.68,95 \%$ CI 0.43 to 1.09$)$ and having an innovative organisational climate $(\mathrm{aRR}=0.66,95 \%$ CI 0.40 to 1.13) were factors with relative high RR estimates, but did not reach statistical significance in the hurdle model. See table 2.

\section{The potential confounding effect of previous history of sick leave}

In the subsample without sick leave, $54 \%$ had sick leave prior to the event. For those with sick leave, the corresponding percentage before the attack was $85 \%$. The aOR of predictability at work was 0.65 (95\% CI 0.41 to $1.05)$ and the aRR of control at work was 0.58 (95\% CI 0.35 to 0.96 ). The estimates became more unstable due to reduced number of cases when adjusting for more covariates. However, our main findings persisted after adjustment for this new covariate.

\section{DISCUSSION \\ Main findings}

The present study explores whether the psychosocial work environment influences the risk of and duration of sick leave among government employees with PTSD symptoms. Of the 94 participants in our sample who fulfilled the PTSD symptom criteria 10 months after the terror attack, 39 individuals had no sick leave. Participants with sick leave did not differ significantly from participants without sick leave on several relevant background variables; one exception was a larger proportion of women in the former group. This is in line with previous research where the terrorist attack had a larger negative impact on the work ability of women. ${ }^{7}$ The PTSD symptoms were also higher among participants with sick leave. As a result, sex and PCL scores were considered as potential confounders and were adjusted for in the analysis. When it comes to psychosocial work environment, both predictability and control in the workplace were important for sick leave.

\section{Interpretation of our findings}

Many employees were engaged in their work even when they had PTSD symptoms. Having a strong attachment 
Table 2 Association between the psychosocial work environment and risk of sick leave among 94 ministerial employees with symptom-defined PTSD, 10 months after a workplace terrorist attack on 22 July in Oslo, 2011

\begin{tabular}{|c|c|c|c|c|}
\hline \multirow[b]{2}{*}{ Psychosocial work factors } & \multicolumn{2}{|c|}{ Binary logistic model } & \multicolumn{2}{|c|}{ Negative binomial count model } \\
\hline & OR (95\% Cl) & aOR $(95 \% \mathrm{Cl}) \dagger$ & RR (95\% Cl) & aRR $(95 \% \mathrm{Cl}) \dagger$ \\
\hline Support from superior & $\begin{array}{l}0.86 \\
(0.55 \text { to } 1.33)\end{array}$ & $\begin{array}{l}0.89 \\
(0.55 \text { to } 1.43)\end{array}$ & $\begin{array}{l}0.80 \\
(0.56 \text { to } 1.14)\end{array}$ & $\begin{array}{l}0.77 \\
\text { (0.53 to } 1.12)\end{array}$ \\
\hline Support from coworkers & $\begin{array}{l}1.12 \\
(0.70 \text { to } 1.81)\end{array}$ & $\begin{array}{l}0.98 \\
(0.58 \text { to } 1.65)\end{array}$ & $\begin{array}{l}0.94 \\
(0.60 \text { to } 1.49)\end{array}$ & $\begin{array}{l}1.00 \\
(0.62 \text { to } 1.62)\end{array}$ \\
\hline Predictability at work & $\begin{array}{l}0.73 \\
(0.49 \text { to } 1.08)\end{array}$ & $\begin{array}{l}0.62^{*} \\
(0.40 \text { to } 0.98)\end{array}$ & $\begin{array}{l}1.10 \\
(0.81 \text { to } 1.49)\end{array}$ & $\begin{array}{l}1.13 \\
\text { (0.84 to } 1.53)\end{array}$ \\
\hline Control of decision & $\begin{array}{l}0.65 \\
(0.33 \text { to } 1.28)\end{array}$ & $\begin{array}{l}0.65 \\
(0.31 \text { to } 1.37)\end{array}$ & $\begin{array}{l}0.65 \\
(0.40 \text { to } 1.05)\end{array}$ & $\begin{array}{l}0.61^{*} \\
(0.38 \text { to } 0.98)\end{array}$ \\
\hline Control of work intensity & $\begin{array}{l}0.75 \\
(0.40 \text { to } 1.42)\end{array}$ & $\begin{array}{l}0.74 \\
(0.37 \text { to } 1.46)\end{array}$ & $\begin{array}{l}0.92 \\
(0.55 \text { to } 1.54)\end{array}$ & $\begin{array}{l}0.88 \\
(0.54 \text { to } 1.43)\end{array}$ \\
\hline Positive challenge at work & $\begin{array}{l}0.99 \\
(0.60 \text { to } 1.66)\end{array}$ & $\begin{array}{l}0.99 \\
(0.56 \text { to } 1.75)\end{array}$ & $\begin{array}{l}0.70 \\
(0.34 \text { to } 1.44)\end{array}$ & $\begin{array}{l}0.82 \\
(0.39 \text { to } 1.69)\end{array}$ \\
\hline Decisional demands & $\begin{array}{l}0.90 \\
(0.50 \text { to } 1.69)\end{array}$ & $\begin{array}{l}0.92 \\
(0.48 \text { to } 1.79)\end{array}$ & $\begin{array}{l}0.86 \\
(0.54 \text { to } 1.36)\end{array}$ & $\begin{array}{l}0.93 \\
(0.56 \text { to } 1.55)\end{array}$ \\
\hline Learning demands & $\begin{array}{l}1.73 \\
(0.88 \text { to } 3.40)\end{array}$ & $\begin{array}{l}1.73 \\
(0.88 \text { to } 3.40)\end{array}$ & $\begin{array}{l}1.23 \\
\text { (0.62 to } 2.45)\end{array}$ & $\begin{array}{l}1.23 \\
(0.62 \text { to } 2.45)\end{array}$ \\
\hline Social organisational climate & $\begin{array}{l}1.39 \\
(0.74 \text { to } 2.62)\end{array}$ & $\begin{array}{l}1.35 \\
(0.69 \text { to } 2.63)\end{array}$ & $\begin{array}{l}0.71 \\
(0.34 \text { to } 1.47)\end{array}$ & $\begin{array}{l}0.71 \\
(0.34 \text { to } 1.47)\end{array}$ \\
\hline Innovative organisational climate & $\begin{array}{l}0.83 \\
(0.47 \text { to } 1.47)\end{array}$ & $\begin{array}{l}0.76 \\
(0.41 \text { to } 1.46)\end{array}$ & $\begin{array}{l}0.69 \\
(0.42 \text { to } 1.11)\end{array}$ & $\begin{array}{l}0.68 \\
(0.43 \text { to } 1.09)\end{array}$ \\
\hline Human resource primacy climate & $\begin{array}{l}0.83 \\
(0.49 \text { to } 1.39)\end{array}$ & $\begin{array}{l}0.76 \\
(0.43 \text { to } 1.36)\end{array}$ & $\begin{array}{l}0.69 \\
(0.43 \text { to } 1.12)\end{array}$ & $\begin{array}{l}0.66 \\
(0.40 \text { to } 1.13)\end{array}$ \\
\hline
\end{tabular}

${ }^{*} \mathrm{P}<0.05$.

†OR and RR adjusted for sex and Post-traumatic Stress Disorder Checklist score (Post-traumatic Stress Disorder Checklist-Specific). aOR, adjusted OR; aRR, adjusted rate ratio; PTSD, post-traumatic stress disorder; RR, rate ratio.

to the workplace could be a constructive coping mechanism after a trauma, emphasising control and empowerment in life. ${ }^{30}$ A positive effect of predictability is in line with previous studies demonstrating that lack of predictability at work, including insufficient information about decisions, future developments and work changes, may increase the risk of burnout, ${ }^{31}$ cognitive stress reactions, ${ }^{32}$ depression $^{33}$ and work absence. ${ }^{34}$

The finding that employees' sense of control was associated with reduced sick leave days corresponds with previous theories on the importance of external and internal control. High sense of control is the belief that one can influence one's situation by efforts and actions, and is a significant factor for increased well-being, enhanced stress management and improved work ability. ${ }^{9-12}$ In addition, the degree of control over one's work situation is associated with high social status, ${ }^{12} 3536$ and the study sample was highly educated with flexible jobs, which is associated with lower sick leave rates. ${ }^{37}$ This can explain why self-reported sick leave after the WTC attack was low since the study participants were employees with a particularly high degree of coping and status.
The present study suggests that an organisation's management should be aware of employees experiencing low predictability and control in their workplace. If feasible, organisations can benefit from increasing employees' work predictability and facilitate proper decision autonomy. These efforts may contribute to a rapid return to work, restore daily routines and normality and keep employees longer at work when sudden major negative events occur, even when employees suffer from psychological distress. However, a prevention strategy focusing merely on the organisational efforts to improve the objective psychosocial work conditions may deal with only part of the problem. Self-reported appraisals of the work environment are not merely based on objective conditions in the workplace, but influenced by individual personality traits and emotions. ${ }^{38} 39$ Our sample of individuals with PTSD and sick leave might therefore appraise their job more negatively and might be less resilient in coping with their symptoms, particularly as negative thoughts of adverse outcomes often accompany psychological distress and symptoms of PTSD. Therefore, together with improved working conditions, a targeted 
therapeutic intervention based on coping and stress management could be appropriate to alter appraisals and even reduce sickness absence.

\section{Strengths and limitations}

The study comprises a unique set of data that allows us to assess how the psychosocial work environment influences the risk of sick leave in the aftermath of a traumatic event. From a relative large cohort of participants, we sampled a population of individuals with symptom-defined PTSD and controlled for PTSD severity. By means of a prospective design with objective registry data on sick leave, we were able to extend our knowledge of the complexity of sick leave risk and how it relates to the psychosocial work environment of people with PTSD symptoms.

The study has several limitations. First, a small sample size of individuals with symptom-defined PTSD reduces the statistical power. Because of this, we may have underestimated the effect of the psychosocial work environment. For example, we found no significant effects of organisational culture. However, an association cannot entirely be ruled out since the effect estimates are high and the CIs are wide and marginally crosses 1 . This goes mainly for the impact of work environments with an innovative climate and good human resource primacy. It is likely that these factors would reach statistical significance in a larger sample and could therefore be important factors in reducing sick leave risk. Second, PTSD diagnosis was based on self-reported measures and may be influenced by social desirability, under-reporting, recall bias and other response bias. However, the Norwegian version of PCL-S has previously been validated as an effective instrument for PTSD screening by the use of self-reported data. ${ }^{20}$ Third, the study population consisted of a majority of highly educated government officials and bureaucrats, and our findings may not necessarily be generalised to other populations. As high education is generally associated with lower rates of sick leave, this could lead to an underestimation of sick leave rates. Finally, another factor reducing the sick leave rates is that extensive acute medical and psychological care was provided by local emergency departments and hospitals following the bombing. Five weeks after the attack, employees were offered an intervention and a follow-up programme by the Occupational Health Service. The psychosocial intervention programme was prioritised to directly exposed individuals, but was accessible by all employees. ${ }^{40}$ In addition, postdisaster leadership training was implemented. These efforts made it possible for employees to return to work.

\section{Implications}

In conclusion, these results suggest that for those suffering from psychological distress following a traumatic event, high sense of control and predictability at work might reduce the sick leave risk. This makes it conceivable for organisations to keep employees working by facilitating optimal working conditions, following terrorism or other major disasters. Further, therapeutic cognitive interventions can alter individuals' negative and worrying appraisals of the working conditions and increase employees' coping skills. These findings are relevant to practitioners seeking to understand the complex relationships between a traumatic event, psychosocial work environment, individual psychological reactions and sick leave risk.

Future research should therefore investigate whether the association is causal and if these findings can be replicated in other populations. As caution is required in interpreting the effects of organisational culture given the limited sample size in this study, further studies should investigate these predictors.

Acknowledgements We thank all the governmental employees who took part in this study.

Contributors TH designed the study. MTGD and AN analysed the data. MTGD drafted the paper. TH, MTGD, AN and MB participated in project meetings where the analysis plan and data interpretation were discussed, and where the article was critically examined and revised.

Funding This work was funded by grants from Research Council Norway (grant number 227 039).

Competing interests None declared.

Patient consent for publication Not required.

Ethics approval Informed consent was obtained from all participants, and the Regional Committee for Ethics in Medical Research approved the study (reference number: 2011/1577).

Provenance and peer review Not commissioned; externally peer reviewed.

Data availability statement Data are available upon reasonable request. The datasets generated during and/or analysed during the current study are not publicly available due to confidentiality agreements made with participants, but are available from the corresponding author on reasonable request.

Open access This is an open access article distributed in accordance with the Creative Commons Attribution Non Commercial (CC BY-NC 4.0) license, which permits others to distribute, remix, adapt, build upon this work non-commercially, and license their derivative works on different terms, provided the original work is properly cited, appropriate credit is given, any changes made indicated, and the use is non-commercial. See: http://creativecommons.org/licenses/by-nc/4.0/.

\section{ORCID iDs}

Maria Teresa Grønning Dale http://orcid.org/0000-0002-0972-2996

Alexander Nissen http://orcid.org/0000-0003-2879-0457

\section{REFERENCES}

1 Boles M, Pelletier B, Lynch W. The relationship between health risks and work productivity. J Occup Environ Med 2004;46:737-45.

2 Clarner A, Graessel E, Scholz J, et al. Work-Related posttraumatic stress disorder (PTSD) and other emotional diseases as consequence of traumatic events in public transportation: a systematic review. Int Arch Occup Environ Health 2015;88:549-64.

3 Stergiopoulos E, Cimo A, Cheng C, et al. Interventions to improve work outcomes in work-related PTSD: a systematic review. BMC Public Health 2011;11:838.

4 APA. Diagnostic and statistical manual of mental disorders. Washington: American Psychiatric Association, 2013.

5 Glynn SM, Drebing C, Penk W. Psychosocial rehabilitation. effective treatments for PTSD: practice guidelines from the International Society for traumatic stress studies. New York: Guilford Press, 2009.

6 Osinubi OYO, Gandhi SK, Ohman-Strickland P, et al. Organizational factors and office Workers' health after the world Trade center terrorist attacks: long-term physical symptoms, psychological distress, and work productivity. J Occup Environ Med 2008;50:112-25. 
7 Hansen MB, Berthelsen M, Nissen A, et al. Sick leave before and after a work-place targeted terror attack. Int Arch Occup Environ Health 2019;92:327-35

8 Birkeland MS, Nielsen MB, Knardahl S, et al. Associations between work environment and psychological distress after a workplace terro attack: the importance of role expectations, predictability and leader support. PLoS One 2015;10:e0119492.

9 Michie S, Williams S. Reducing work related psychological ill health and sickness absence: a systematic literature review. Occup Environ Med 2003:60:3-9.

10 Vahtera J, Kivimäki M, Pentti J. Effect of change in the psychosocial work environment on sickness absence: a seven year follow up of initially healthy employees. J Epidemiol Community Health 2000;54:484-93.

11 TWH N, Sorensen KL, Eby LT. Locus of control at work: a metaanalysis. J Organ Behav 2006;27:1057-87.

12 North F, Syme SL, Feeney A, et al. Explaining socioeconomic differences in sickness absence: the Whitehall II study. BMJ 1993;306:361-6.

13 Hansen MB, Birkeland MS, Nissen A, et al. Prevalence and course of Symptom-Defined PTSD in individuals directly or indirectly exposed to terror: a longitudinal study. Psychiatry 2017;80:171-83.

$14 \varnothing \mathrm{S}$, Blix I, Heir T. The aftermath of terrorism: posttraumatic stress and functional impairment after the 2011 Oslo bombing. Front Psychol 2015;6:1156-56.

15 Schuster MA, Stein BD, Jaycox LH, et al. A national survey of stress reactions after the September 11, 2001, terrorist attacks. $N$ Engl J Med 2001;345:1507-12.

16 Heir T, Blix I, Knatten CK. Thinking that one's life was in danger: perceived life threat in individuals directly or indirectly exposed to terror. Br J Psychiatry 2016;209:306-10.

17 Ehlers A, Clark DM. A cognitive model of posttraumatic stress disorder. Behav Res Ther 2000;38:319-45.

18 Nissen A, Birkeland Nielsen M, Solberg Øivind, et al. Perception of threat and safety at work among employees in the Norwegian ministries after the 2011 Oslo bombing. Anxiety, Stress, \& Coping 2015;28:650-62.

19 Weathers FW LB, Herman DS, Huska JA, et al. The PTSD checklist $(P C L)$ : reliability, validity, and diagnostic utility. San Antonio, Tex: International Society of Traumatic Stress Studies, 1993.

20 Hem C, Hussain A, Wentzel-Larsen T, et al. The Norwegian version of the PTSD checklist (PCL): construct validity in a community sample of 2004 tsunami survivors. Nord J Psychiatry 2012;66:355-9.

21 American Psychiatric Association. Diagnostic and statistical manual of mental disorders, DSM-IV. Washington, DC: American Psychiatric Association, 1994.

22 Dallner M EA-L, Gamberale F, Hottinen V, et al. Validation of the general Nordic questionnaire (QPSNordic) for psychological and social factors at work. Copenhagen: Nordic Council of Ministers, 2000.

23 Lindström K, Ørhede E, Elo A-L, et al. User's guide for the QPSNordic: general Nordic questionnaire for psychological and social factors at work. Copenhagen: Nordic Council of Ministers, 2000.
24 Strand BH, Dalgard OS, Tambs K, et al. Measuring the mental health status of the Norwegian population: a comparison of the instruments SCL-25, SCL-10, SCL-5 and MHI-5 (SF-36). Nord J Psychiatry 2003;57:113-8.

25 Berntsen D, Rubin DC. The centrality of event scale: a measure of integrating a trauma into one's identity and its relation to post-traumatic stress disorder symptoms. Behav Res Ther 2006;44:219-31.

26 Michalos AC. Cantril's Ladder. In: Encyclopedia of quality of life and well-being research. Springer Netherlands: Dordrecht, 2014.

27 Elklit A, Schmidt Pedersen S, Jind L. The crisis support scale: psychometric qualities and further validation. Pers Individ Dif 2001;31:1291-302.

28 Cameron AC, Trivedi PK. Regression analysis of count data. Second ed. Cambridge, UK: Cambridge University Press, 2013.

29 Rodriguez G. Models for count data with overdispersion. retrieved from Princeton university, 2013. Available: http://dataprincetonedu/ wws509/notes/c4apdf

30 Luthar SS, Cicchetti D, Becker B. The construct of resilience: a critical evaluation and guidelines for future work. Child Dev 2000;71:543-62.

31 Borritz M, Rugulies R, Christensen KB. Burnout as a predictor of self-reported sickness absence among human service workers: prospective findings from three year follow up of the PUMA study. Occup Environ Med 2006;63:98-106.

32 Albertsen K, Rugulies R, Garde AH, et al. The effect of the work environment and performance-based self-esteem on cognitive stress symptoms among Danish knowledge workers. Scand J Public Health 2010;38:81-9.

33 Netterstrøm B, Conrad N, Bech P, et al. The relation between workrelated psychosocial factors and the development of depression. Epidemiol Rev 2008;30:118-32.

34 Nielsen ML, Rugulies R, Christensen KB, et al. Psychosocial work environment predictors of short and long spells of registered sickness absence during a 2-year follow up. J Occup Environ Med 2006;48:591-8.

35 Griffin JM, Fuhrer R, Stansfeld SA, et al. The importance of low control at work and home on depression and anxiety: do these effects vary by gender and social class? Soc Sci Med 2002;54:783-98.

36 Siegrist J. Chronic psychosocial stress at work and risk of depression: evidence from prospective studies. Eur Arch Psychiatry Clin Neurosci 2008;258 Suppl 5:115-9.

37 Robroek SJW, van Lenthe FJ, Burdorf A. The role of lifestyle, health, and work in educational inequalities in sick leave and productivity loss at work. Int Arch Occup Environ Health 2013;86:619-27.

38 Wainwright D, Calnan M. Work stress: the making of a modern epidemic. Open University Press, 2002.

39 Wainwright D, Calnan M. The fall of work stress and the rise of wellbeing. In: Vickerstaff S, Phillipson C, Wilkie R, eds. Work, health and wellbeing: the challenges of managing health at work. Bristol: Policy Press, 2012

40 Weisæth L, Heir T. Workplace and organizational disasters: response and planning. In: Ursano R, ed. Textbook of disaster psychiatry. Cambridge: Cambridge University Press, 2017. 